\title{
Sliding Mode Anti-windup Strategy for Mass Flow-Rate Regulation of Compressors with Rate-Limited Motors
}

\author{
Yousif Eldigair, Cristian Kunusch, Carlos Ocampo-Martinez, Pablo Camocardi
}

\begin{abstract}
In this paper, the control of the mass flow-rate for a centrifugal compressor is discussed. The compressor is driven by a rate-limited actuating motor. To avoid integral windup, a sliding mode control (SMC) based anti-windup strategy is proposed. The developed scheme essentially avoids the integral windup of PI controllers by adapting the controller gain such that output of controller is not influenced by the rate limiter. Furthermore, a relay-based optimal tuning methodology for PI controllers in rate-limited systems is presented and discussed. Optimal tuning of the PI controller is done at different operating conditions of the compressor and used to create a gain schedule that provides a more robust control structure compared to standard manually tuned PI controllers.
\end{abstract}

\section{INTRODUCTION}

When implementing PI control strategies on physical systems, constraints that exists due to limitations in the actuators are generally encountered. Such constraints can often be problematic as they lead to the integral windup of the PI controller. In response, many different variations of antiwindup (AW) strategies have been proposed by researchers to tackle this issue. Most of these AW strategies [1], [2] are directed towards actuators with a saturation, as seen in valves for example, with some published works presenting detailed proof of stability and performance [3]-[5]. A less common form of actuator constraints that can also lead to integral windup is slew rates generally imposed by rate limiters. This can be seen in such plants involving hydraulic actuators, electric motors, compression and aircraft systems for example, where fast transients can essentially damage the system. Coping with this type of constraints is the prime focus of this paper.

While imposing a rate limit might increase the lifetime of some actuators due to softer transients, the controller windup caused by rate limiters generally leads to poor control performance or, in extreme case, may result in instablity. A number of windup strategies were devised to overcome this issue. These AW schemes can generally be classified into two groups [6]. The first class includes the conventional AW methods where the controller is modified, usually the integral term, such that the windup is suppressed. In the second class, the reference signal to the control system is conditioned as to respect the physical constraints of the process input. This can be done by either using low pass filters or rate limiters at the reference. More effectively however, a signal can be injected and added to the reference such that the controller

Yousif Eldigair, Cristian Kunusch and Pablo Camocardi are with Brose Fahrzuegteile GmbH \& Co. Kommanditgesellschaft, Würzburg, Germany.

Carlos Ocampo-Martinez is with Universitat Politècnica de Catalunya, Institut de Robòtica i Informàtica Industrial, CSIC-UPC, Barcelona, Spain. output does not violate the rate limit constraint. In [6], [7], a sliding mode controller (SMC) was used to generate this signal; thereby, conditioning the reference.

The presence of rate limiters in closed-loop systems adds complexity to the tuning process of PI controllers. This is due to the fact that, unlike magnitude saturation, rate limiters display dynamic properties that can mask the dynamics of the actual plant. Relay-based tuning methods such as the Anstrom-Hagglund relay feedback test (RFT), explained in [8]-[10], would therefore be severely impacted by the phase shift and attenuation introduced by rate limiters. As such, it is essential to study the dynamics of rate limiters and determine their impacts on the oscillations when using relaybased tuning methods.

In this paper, the control of the mass-flow for a centrifugal air compressor driven by a rate-limited electric motor is considered. To mitigate the windup effect caused by the rate limiter, a flexible anti-windup method is proposed which can be retrofitted in a straightforward manner with minimal knowledge of the system regardless of the rate-limit or system parameters. It features SMC used to adjust the gain of the PI controller such that the output of the controller abides the slew rate set by the rate limiter. Furthermore, in this paper, a methodology of relay-based tuning of PI controllers in rate-limited systems is discussed. Analysis is done via the describing functions method. Due to the nonlinearity of the compression system, the tuning was done at different operating conditions to generate a gain schedule for the controller gain. The end result is a robust PI controller that does not suffer anti-windup during fast transients and operates optimally when the rate limiter is not in effect.

In Section II of this paper, an overview of the compression system, the major control loop and the models associated is presented. Section III presents the sliding mode AW scheme used to mitigate integral anti-windup. The procedure followed to develop a robust PI controller is then explained in Section IV. Section V presents the simulation results of the developed strategies. Finally, some conclusions are drawn in Section VI.

\section{AIR COMPRESSION SYSTEM WITH RATE LIMITER}

In some fuel-cell systems, oxygen is taken from the atmosphere through a compressor and is delivered to a fuel-cell stack where it reacts with hydrogen to produce electricity and water [11]. For a particular load on the fuelcell, a certain mass flow-rate of air, or oxygen, is demanded. Supplying lower flow-rates than is required would lead to 


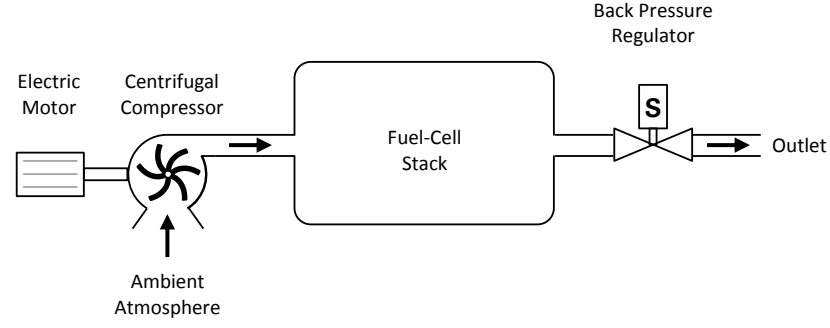

Fig. 1. Conceptual scheme of the compression system

oxygen starvation which limits the fuel-cell operation while supplying a higher flow-rate diminishes the overall efficiency of the system by overworking the compressor. Accordingly, the mass flow-rate of air into the stack must be controlled and set to track a particular reference which is determined by the load current on the fuel-cell. Moreover, the fuel-cell stack can be made to operate at different pressure conditions. This in turn can enhance the efficiency of the system. Regulation of both pressure and mass flow of air into the cathode of stack can be achieved by the compression system shown in Fig. 1.

In the system shown in Fig. 1, the pressure is controlled via a fast acting back-pressure regulator valve. The control is done in such a manner that the pressure can be assumed constant at any particular operating point. Focus in this paper is given to the control of the mass flow-rate, $W_{c p}$ (in $\mathrm{kg} / \mathrm{s}$ ), using the centrifugal compressor. This is to be achieved through varying the speed of rotation of the compressor as depicted by the block diagram shown in Fig. 2 .

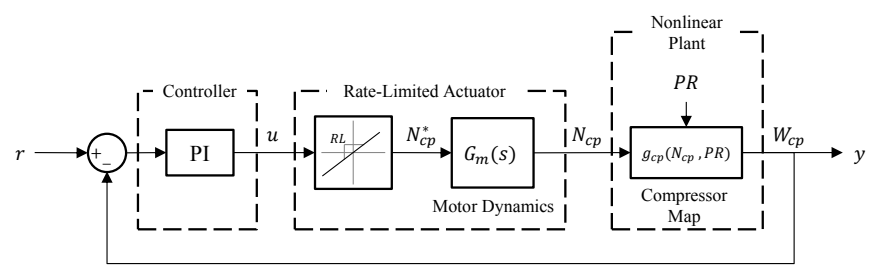

Fig. 2. Block diagram of flow-rate control loop

In the considered system, the centrifugal air compressor is driven by an electric motor. As to prevent high current transients and reduce mechanical stresses on the compressor, the reference speed to the motor, $N_{c p}^{*}$ (in rpm), is limited by a rate limiter. The dynamics of a non-salient three phase permanent magnet synchronous motor (PMSM) can be modeled by

$$
\begin{aligned}
\frac{\mathrm{d} i_{d}}{\mathrm{~d} t} & =\frac{1}{L s}\left(v_{d}-R_{s} i_{d}+\left(\frac{P}{2}\right) \omega_{c p} L_{s} i_{q}\right) \\
\frac{\mathrm{d} i_{q}}{\mathrm{~d} t} & =\frac{1}{L s}\left(v_{q}-R_{s} i_{q}-\left(\frac{P}{2}\right) \omega_{c p}\left(\Psi_{f}+L_{s} i_{d}\right)\right), \\
\frac{\mathrm{d} \omega_{c p}}{\mathrm{~d} t} & =\frac{1}{J}\left(T_{e}-T_{l}\left(W_{c p}, N_{c p}\right)-B_{m} \omega_{c p}\right) \\
T_{e} & =\left(\frac{P}{3}\right) \Psi_{f} i_{q}
\end{aligned}
$$

where

$\begin{array}{lll}i_{d}, i_{q} & d q \text {-stator currents } & (\mathrm{A}) \\ v_{d}, v_{q} & d q \text {-stator voltages } & (\mathrm{V}) \\ R_{s} & \text { Stator resistance } & (\Omega) \\ L_{s} & \text { Stator inductance } & (\mathrm{H}) \\ \Psi_{f} & \text { Magnet flux linkage } & (\mathrm{Wb}) \\ \omega_{c p} & \text { Rotational speed } & (\mathrm{rad} / \mathrm{s}) \\ N_{c p} & \text { Rotational speed } & (\mathrm{rpm}) \\ T_{e} & \text { Electromagnetic torque } & (\mathrm{N} \cdot \mathrm{m}) \\ T_{l} & \text { Load torque } & (\mathrm{N} \cdot \mathrm{m}) \\ J & \text { Moment of inertia } & \left(\mathrm{kg} \cdot \mathrm{m}^{2}\right) \\ B_{m} & \text { Drag coefficient } & (\mathrm{N} \cdot \mathrm{m} \cdot \mathrm{s} / \mathrm{rad}) \\ P & \text { Number of poles } & (-)\end{array}$

The speed in rpm can be calculated as $N_{c p}=\frac{30}{\pi} \omega_{c p}$. The standard field oriented control (FOC) method using PI controllers is applied to track the reference speed, $N_{c p}^{*}$. The load torque $T_{l}\left(W_{c p}, N_{c p}\right)$ is the torque demanded by the compression process. It is a nonlinear function which depends on the speed of rotation, $N_{c p}$, and the output mass flow-rate $W_{c p}$. For a turbo compressor, this mass flow-rate is determined by the pressure ratio, $P R$, from inlet to outlet and the speed, $N_{c p}$. Both $T_{l}\left(W_{c p}, N_{c p}\right)$ and $W_{c p}$ are generally described by static maps such as the ones shown in Fig. 3 and Fig. 4.

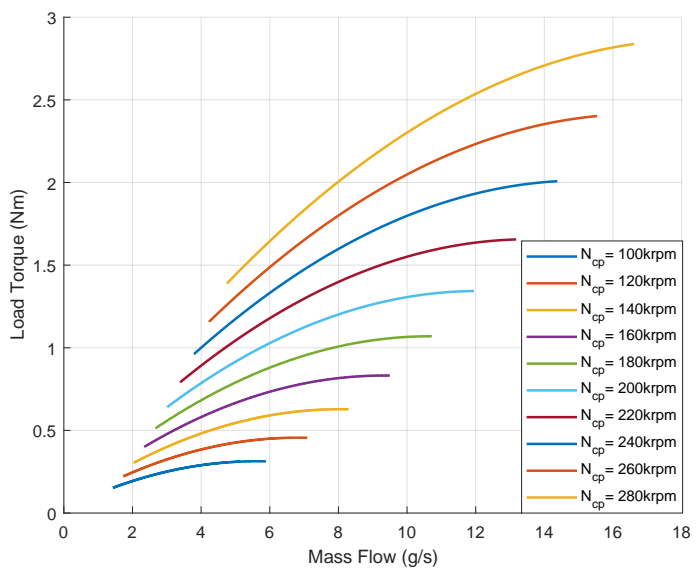

Fig. 3. Compression torque map

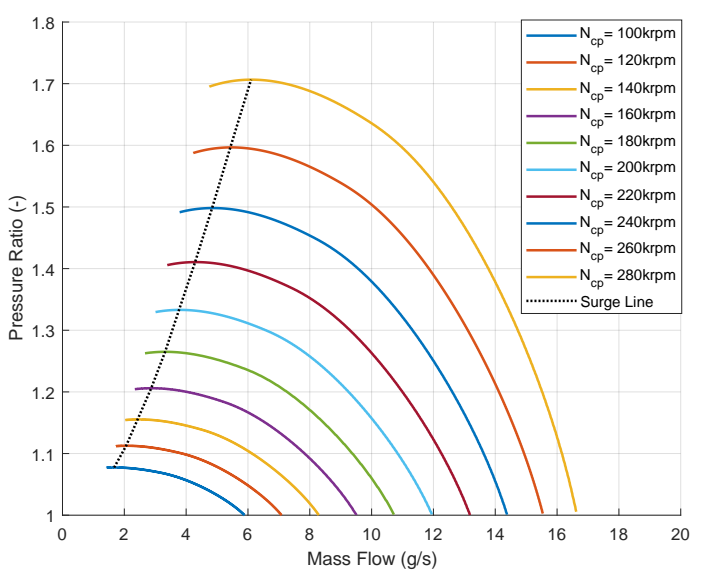

Fig. 4. Compressor mass flow map 
It should be stressed that if the mass flow is insufficient for a particular pressure ratio, the compressor experiences an instability known as surge during which high oscillations in the flow occur and vigorous vibrations are generated. This consequently results in severe damage to the compressor. Hence, the compressor is generally operated in the stable region right of the surge line. Surge can occur due sudden changes in production which lead to a fast pressure rise. Another cause of surge is the rapid deceleration of the motor. One precaution which is taken to prevent this is to limit the deceleration rate of the electric motor. As such, a negative rate limit of $\mathrm{RL}^{-}=-50 \mathrm{krpm} / \mathrm{s}$ is implemented while the positive rate limit is kept higher at $\mathrm{RL}^{+}=150 \mathrm{krpm} / \mathrm{s}$.

Due to the presence of an asymmetric rate limiter $\left(\left|R L^{+}\right| \neq\left|R L^{-}\right|\right)$in the control loop and the nonlinearity introduced by the compressor map, the implementation of conventional AW techniques may not successfully mitigate integral windup leading to output overshoots or may just result in an unnecessary slow convergence response. As such, for the considered compression system, a more robust AW strategy must be adopted.

\section{SLIDING MODE ANTI-WINDUP SCHEME}

To prevent integral windup in PI controllers in rate-limited systems, a sliding mode controller (SMC) can be utilized. This SMC can be used to adapt the gain of the PI controller such that its output tracks the output of the rate limiter. The structure shown in Fig. 5 is therefore proposed. Henceforth, this method of anti-windup will be referred to as sliding mode adaptive gain (SMAG).

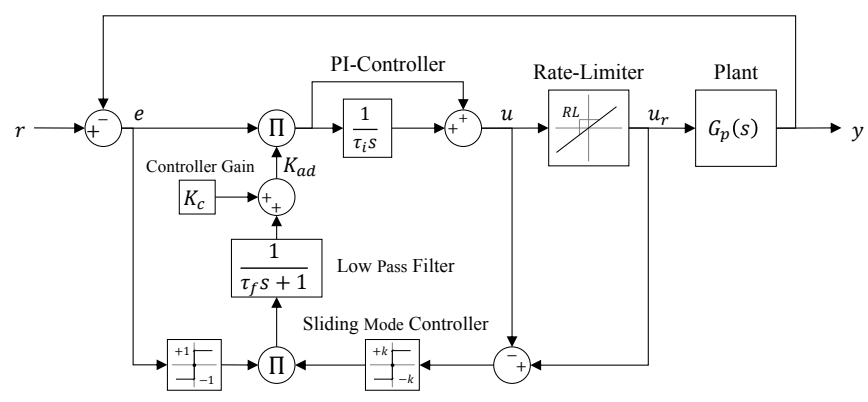

Fig. 5. Block diagram of sliding mode adaptive gain (SMAG) anti-windup

The SMC is used in its standard form given by the control law [12]

$$
u_{\mathrm{smc}}=k \operatorname{sign}(\sigma)=\left\{\begin{array}{cll}
k & \text { if } & \sigma>0 \\
-k & \text { if } & \sigma<0 \\
0 & \text { if } & \sigma=0
\end{array}\right.
$$

where $k$ is a positive real number and the sliding variable $\sigma$ is defined as

$$
\sigma=u_{r}-u \text {. }
$$

Here, $u$ is the output of the PI controller and $u_{r}$ is the ratelimited input to the plant as seen in Fig. 5. For proper SMC operation, the reachability condition given by $\dot{\sigma} \sigma<-\eta|\sigma|$ must be satisfied [12]. Here, $\eta$ is a positive number that determines the reaching time during the convergence of $\sigma$.
Since the relative degree of $\sigma$ is one, this can be achieved using the standard form given in (5).

To prove that the reachability condition holds for all values of $\sigma$, the derivative $\dot{\sigma}$ is first evaluated as

$$
\begin{aligned}
\dot{\sigma} & =\frac{\mathrm{d} u_{r}}{\mathrm{~d} t}-\frac{\mathrm{d}}{\mathrm{d} t}\left(e K_{a d}+\frac{1}{\tau_{i}} \int e K_{a d} \mathrm{~d} t\right), \\
& =\frac{\mathrm{d} u_{r}}{\mathrm{~d} t}-\left(\dot{e} K_{a d}+e \dot{K}_{a d}+\frac{1}{\tau_{i}} e K_{a d}\right),
\end{aligned}
$$

where $e=y-r$ is the output reference tracking error, $\tau_{i}$ is the integral time constant of the PI-controller and $K_{a d}$ is the adapted gain of the PI controller to avoid integral windup. The derivative of of $K_{a d}$ can be expressed as

$$
\frac{\mathrm{d} K_{a d}}{\mathrm{~d} t}=\frac{1}{\tau_{f}} K_{c}(1+k \operatorname{sign}(\sigma e)),
$$

where $\tau_{f}$ is the time constant of the low pass filter used. Substituting (8) into (7) yields

$$
\dot{\sigma}=\frac{\mathrm{d} u_{r}}{\mathrm{~d} t}-\dot{e} K_{a d}-\frac{1}{\tau_{f}}|e| k \operatorname{sign}(\sigma)+\left(\frac{1}{\tau_{f}}-\frac{1}{\tau_{i}}\right) e K_{a d} .
$$

It should be noted that the derivative $\frac{\mathrm{d} u_{r}}{\mathrm{~d} t}$ is restricted by the rate limiter and can therefore be expressed as

$$
\frac{\mathrm{d} u_{r}}{\mathrm{~d} t}=\left\{\begin{array}{cc}
\mathrm{RL}^{-} & \text {if } \quad \sigma>0, \\
\mathrm{RL}^{+} & \text {if } \quad \sigma<0, \\
\frac{\mathrm{d} u}{\mathrm{~d} t} & \text { if } \quad \sigma=0,
\end{array}\right.
$$

where $\mathrm{RL}^{-}$and $\mathrm{RL}^{+}$are negative and positive constants representing the slew rates. If the rate limiter is symmetric, then $\left|\mathrm{RL}^{-}\right|=\left|\mathrm{RL}^{+}\right|=\mathrm{RL}$ and $\frac{\mathrm{d} u_{r}}{\mathrm{~d} t}=-\mathrm{RL} \operatorname{sign}(\sigma)$ for $\sigma \neq 0$. Accordingly, the reachability condition can be rewritten as

$$
\dot{\sigma} \sigma=\sigma f-|\sigma| \rho<-\eta|\sigma|
$$

where

$$
\begin{aligned}
& f=-\dot{e} K_{a d}+\left(\frac{1}{\tau_{f}}-\frac{1}{\tau_{i}}\right) e K_{a d}, \\
& \rho=\mathrm{RL}+\frac{1}{\tau_{f}}|e| k .
\end{aligned}
$$

Here, (11) is satisfied if $\rho>f$. Assuming that $\left|\dot{e} K_{a d}\right|$ is bounded such that $\mathrm{RL}>\left|\dot{e} K_{a d}\right|$, there exists a sufficiently large value of $k$ for which $k>\left(1-\frac{\tau_{f}}{\tau_{i}}\right) K_{a d}$. For such a value, $\rho>f$ and (11) is satisfied.

The developed SMAG was first implemented alongside the mass flow control loop shown in Fig. 2 and tested in simulation. The PI control parameters $K_{c}$ and $\tau_{i}$ were initially set to $4.5 \times 10^{6}$ and 0.115 respectively. A filter time constant $\tau_{f}$ of 0.01 was used. The gain $k$ of the SMC was chosen to be equal to $K_{c}$. This is high enough to satisfy the reachability condition.

As illustrated in Fig. 6, the output of the controller $u$ successfully tracks the output of the rate limiter $u_{r}$. This, for a step response, is achieved by the trend of the controller gain shown. The trend is obtained for both a step increase and a step decrease in the reference. The output response shown in Fig. 7 shows the windup phenomenon that is encountered without any AW strategy. Since $\left|R L^{-}\right|<\left|R L^{+}\right|$, a larger windup occurs for a step decrease in the reference than for 

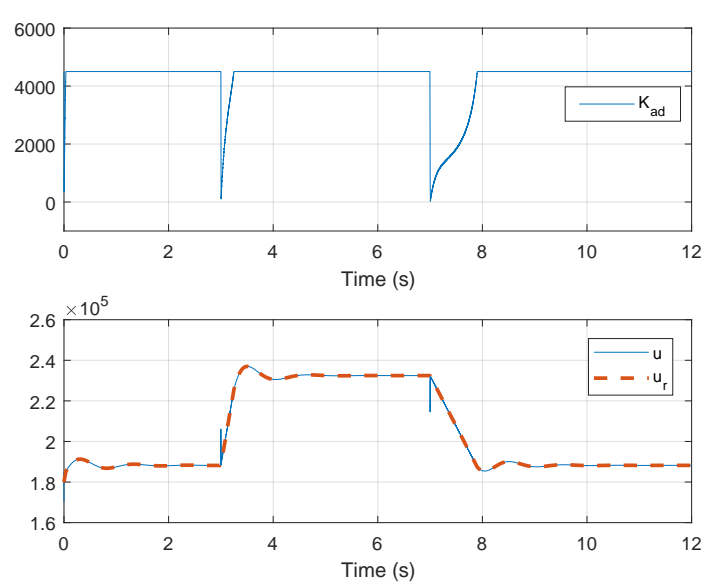

Fig. 6. SMAG anti-windup response to references step changes

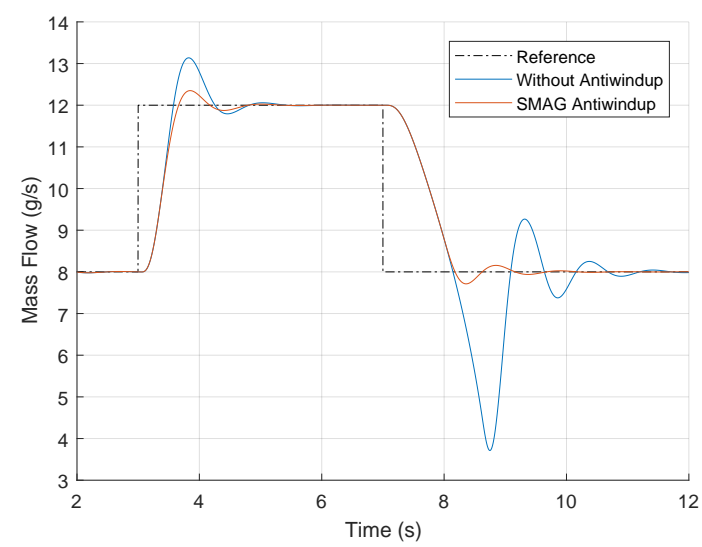

Fig. 7. Output response with and without SMAG anti-windup to reference step changes

a step increase. The developed SMAG does however nullify this effect. During the ramp up/down phase when $\sigma=0$, the PI controller does not windup since the input to the system $u_{r}$ is essentially the same as the controller output $u$. This continues until $K_{a d}=K_{c}$ after which the rate limiter no longer influences the behavior of the system.

\section{TUNING OF PI CONTROLLERS IN RATE-LIMITED SYSTEMS}

In tuning the flow-rate controller of the system in Fig. 1, two challenges are presented. First is that standard tuning methods, such as relay-based tuning methods, cannot be directly applied due the existence of a rate limiter in the control loop which interferes with the tuning process. Therefore, it is critical to analyze the impacts of this rate limiter on tuning methods. This was done using the describing functions method of analysis. Second is that, since the compressor map introduces a nonlinearity, tuning must be done for different operating conditions. A gain schedule can then be setup to increase the robustness of the control approach. Otherwise, parameters obtained for one operating condition might lead to instability in other conditions.

\section{A. Relay Feedback Test of Rate-Limited Systems}

In the standard Astrom-Hagglund relay-feedback test (RFT), oscillations are created at the negative real axis of the complex plane. The resultant frequency and amplitude of the oscillations are then used with tuning laws such as Ziegler-Nichols to calculate PI control parameters. However, if a rate limiter is incorporated, a phase lag is introduced. To counteract this phase lag, the standard RFT can be altered to include an additional derivative term as shown in Fig. 8. The use of a low pass filter with the derivative is unnecessary because the rate-limiter itself acts as a filter and attenuates high frequency components.

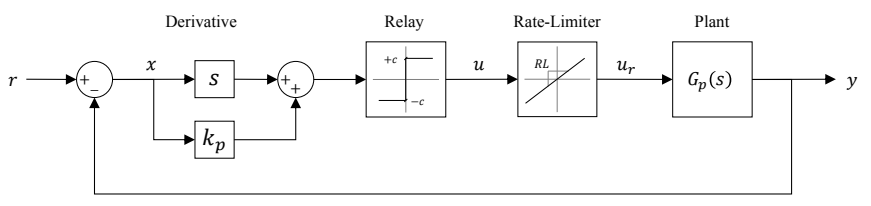

Fig. 8. RFT with additional derivative term in rate-limited system

The RFT presented in Fig. 8 with the additional derivative term can be analyzed via the describing functions (DF) method. Here, the combined nonlinearity of both the relay and rate-limiter along with the gain $k_{p}$ and derivative are all expressed as a complex gain. Since the derivative and rate limiters are essentially dynamic (affected by frequency of $x$ ), the DF, $N(a, \omega)$, will be a function of both amplitude $a$ and frequency $\omega$ of the resultant oscillations. For simplicity, it can be assumed that the rate limiter is symmetric and that the relay output $c$ is large enough such that the rate limiter is always acting.

Consider now the case where the parameter $k_{p}$ of the test, shown in Fig. 8, is quite high such that the derivative term is dropped. This imitates the standard RFT. In this scenario, the DF can be derived as

$$
\begin{aligned}
N(a, \omega) & =j \frac{4}{\pi a} \int_{0}^{\frac{\pi}{2}}\left(\frac{2 a_{r}}{\pi} \psi-a_{r}\right) \cos \psi \mathrm{d} t, \\
& =-j \frac{8}{\pi^{2}} \frac{a_{r}}{a},
\end{aligned}
$$

where $a_{r}$ denotes the amplitude of oscillations of the triangular output signal of the rate limiter. For large values of $c, a_{r}$ is a direct function of the frequency $\omega$ and the slew-rate RL and can be calculated as $a_{r}=\frac{\pi \mathrm{RL}}{2 \omega}$. Accordingly, the negative reciprocal of the describing function can be expressed as

$$
-N^{-1}(a, \omega)=-j \frac{\pi a \omega}{4 \mathrm{RL}} .
$$

The harmonic balance equation (HBE) given

$$
G_{p}\left(j \omega_{0}\right)=-N^{-1}\left(a_{0}, \omega_{0}\right)
$$

can then be solved to find the frequency $\omega_{0}$ and amplitude $a_{0}$ of the excited oscillations. Since $-N^{-1}(a, \omega)$ is a negative imaginary number, if the standard RFT is used without a derivative term, limit cycles are created on the negative imaginary axis. Hence, the standard RFT is not suitable for PI controller tuning. 
Consider now the impact of the additional derivative term. By introducing an additional derivative term to the standard RFT as shown in Fig. 8, limit cycles can be created at an angle $\phi$ from the negative real axis. This is due to the phase shift introduced by the derivative term which can be evaluated as

$$
\phi=\arctan \left(\frac{k_{p}}{\omega}\right) .
$$

It should be noted that the gain of the combined nonlinearity remains the same since the rate limiter essentially imposes a saturation on the rate which is a function of the amplitude $a$ and frequency $\omega$. Accordingly,

$$
|N(a, \omega)|=\left|\frac{4 \mathrm{RL}}{\pi a \omega}\right| .
$$

Through expressing the $N(a, \omega)$ in rectangular coordinates, the describing function with the additional derivative term becomes

$$
\begin{aligned}
N(a, \omega) & =\frac{4 \mathrm{RL}}{\pi a \gamma}\left(1-j \frac{k_{p}}{\omega}\right), \\
-N^{-1}(a, \omega) & =-\frac{\pi a \gamma}{4 \mathrm{RL}}\left(1+j \frac{\omega}{k_{p}}\right),
\end{aligned}
$$

where

$$
\gamma=\sqrt{\omega^{2}+k_{p}^{2}}
$$

To compensate for the $90^{\circ}$ phase shift caused by the rate limiter nonlinearity, the phase $\phi$ is set to zero by selecting a proportional gain $k_{p}$ of zero.

\section{B. Optimal Tuning}

To generate optimal tuning laws for the considered compression system, it is convenient to first obtain a linearized transfer function representation which approximates of the nonlinear process. Most commonly, the first-order plus dead time (FOPDT) transfer function given by $\frac{K e^{-s \theta}}{\tau s+1}$ is used. However, in this work, a second-order plus dead time (SOPDT) given by

$$
G_{p l}(s)=\frac{K_{p l} e^{-s \theta_{p l}}}{\tau_{p l}^{2} s^{2}+2 \xi \tau_{p l} s+1}
$$

is used, which better approximates the dynamics of the compression process. System identification can be done using the RFT with the additional derivative term shown in Fig. 8 by selecting different valves of $k_{p}$ and exciting oscillations at different frequencies. It is important to note that for the considered compression system, the dynamics are primarily introduced by the motor. Through system identification, it can be shown that, throughout all the operating conditions $\left(W_{c p}\right.$ and $P R$ ) the motor response from $N_{c p}^{*}$ to $N_{c p}$ can be approximated by a SOPDT transfer function with the parameters

$$
\tau_{p l}=0.15, \quad \theta_{p l}=0.05, \quad \xi=0.7 .
$$

The motor dynamics impose a gain of one since at steady state $N_{c p}^{*}=N_{c p}$. Furthermore, in a small domain of operating conditions, the compressor map from $N_{c p}$ to $W_{p}$ can be can be represented by

$$
W_{c p}=K_{m a p} N_{c p}+W_{o}
$$

where $W_{o}$ represents a certain bias and $K_{\text {map }}$ is a gain. The bias $W_{o}$ can be considered to be a disturbance and ignored since it does not affect the required tuning parameters.

In [9], homogeneous tuning rules for PID controllers were proposed. In a similar fashion, for systems with rate-limited actuators, the following rules can be used

$$
K_{c}=c_{1} \frac{4 \mathrm{RL}}{\pi a_{0} \omega_{0}}, \quad \tau_{i}=c_{2} \frac{2 \pi}{\omega_{0}} .
$$

Here $c_{1}$ and $c_{2}$ are constants that define the tuning law to be used. The idea behind (23) is to express the control parameters as homogeneous functions of the critical gain $\frac{4 R L}{\pi a_{0} \omega_{0}}$ and the critical period $\frac{2 \pi}{\omega}$ which are defined at an angle $\phi_{c}$ from the negative real axis. This is done to account for the phase shift introduced by the controller. The phase shift $\phi_{c}$ can be evaluated as

$$
\phi_{c}=-\arctan \left(\frac{1}{2 \pi c_{2}}\right) .
$$

The compression system in Fig. 1 can be linearized for a small domain of operating conditions $\left(W_{c p}\right.$ and $\left.P R\right)$ and approximated by (21). For a SOPDT system having $\xi=0.7$ and a ratio $\frac{\theta}{\tau}=\frac{1}{3}$, the optimal tuning to have the minimum integral absolute error (IAE) with a gain margin of 3 is $c_{1}=$ 0.317 and $c_{2}=0.488$. For this tuning rule, the phase shift can be calculated using (24) as $\phi_{c}=-18.0^{\circ}$.

Using the RFT with an additional derivative term as shown in Fig. 8 for a rate-limited system is an iterative process. First, the closed-loop system is allowed to reach steady-state oscillations from which the frequency $\omega_{0}$ is measured and $\phi$ is calculated using (17). The gain $k_{p}$ is then increased or decreased and $\phi$ is recalculated for the new $\omega_{0}$. This process is repeated until the $\phi=-\phi_{c}=-18.0^{\circ}$, at which point the amplitude $a_{0}$ of output oscillations is measured. The parameters of the PI controller are then calculated using (23). This yields the optimal parameters at one operating point in the compressors map and can be repeated for different conditions.

\section{Gain Scheduling}

The proposed RFT was applied at a number of selected operating conditions ( $P R$ and $W_{c p}$ ). It is noteworthy that for the considered simulation model, the system time constants do not change at different points of operation; rather, only the gain of the compressor map varies. Consequently, the value of $\tau_{i}$ does not vary while $K_{c}$ changes with the operating conditions. Applying the proposed RFT yields $\tau_{i}=0.274$. The values of $K_{c}$ obtained for different points on the compressor map are can be used in to set-up a gain schedule for added robustness to the control loop. A smooth approximation can made to fit the data points using

$$
\begin{aligned}
& K_{c}\left(W_{c p}, P R\right)=\left(1.13-11.3 W_{c p}-1.42 P R\right. \\
& \left.\quad-143 W_{c p}^{2}+18.0 P R W_{c p}+0.41 P R^{2}\right) \times 10^{8} .
\end{aligned}
$$




\section{Optimally Tuned Gain Scheduled PI CONTROLLER WITH SMAG}
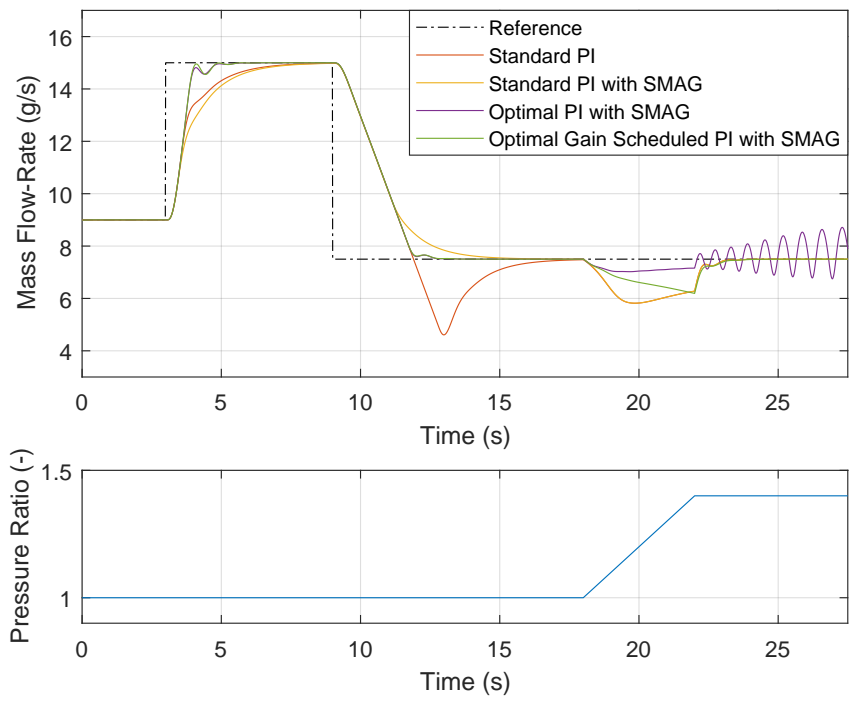

Fig. 9. Response of mass flow-rate for different control strategies

In the considered rate limited system, integral windup can be avoided by selecting a very small controller gain. However, this results in an unnecessarily slow response. Using the proposed SMAG strategy for AW was shown to successfully avoid wind-up when using higher controller gains leading to faster convergence. However, to ensure that the PI controller behaves optimally when the rate limiter is not saturated, it can be tuned using an RFT with an additional derivative term to compensate for the phase lag introduced by the rate limiter. This ensures that a predefined gain margin is obtained and leads to the best performance (lowest IAE) at that margin. This, however, applies for a linear plant. Since a compressor behaviors in a nonlinear fashion, tuning must be carried out at different operating conditions of mass flow and pressure ratio and a gain schedule must be constructed. Not doing so can alter the gain margin or even result in instabilities under different operating conditions, for example, at higher pressure ratios. This shown by the response of the PI controller which was optimized at a low pressure ratio and was not implemented with a gain schedule. It can be seen in Fig. 9 that this controller lead to an instability at higher pressure ratios. The output responses presented in Fig. 9 highlights the need and effects of SMAG, optimal tuning and gain scheduling on the control of the mass flow-rate of the centrifugal compressor.

\section{CONCLUSION}

To cope with systems having rate-limited actuators, the developed SMAG anti-windup strategy can be adopted. This ensures that the PI controller operates at the actuators' rate-limit while avoiding integral windup during transients. The topology is also quite flexible as it does not require knowledge of plant dynamics; rather, only the rate-limit imposed. Furthermore, to tune the PI controller, a systematic method was devised. This method can also be utilized in systems without rate limiters as a more lenient alternative to the standard RFT. By following the tuning procedure discussed in this paper, the optimal control parameters can be obtained for the rate-limited system.

\section{REFERENCES}

[1] G. Herrmann, M. C. Turner, I. Postlethwaite, and G. Guo, "Practical implementation of a novel anti-windup scheme in a HDD-dual-stage servo-system," IEEE/ASME Transactions on Mechatronics, vol. 9, no. 3, pp. 580-592, 2004.

[2] G. Grimm, J. Hatfield, I. Postlethwaite, A. R. Teel, M. C. Turner, and L. Zaccarian, "Antiwindup for stable linear systems with input saturation: an LMI-based synthesis," IEEE Transactions on Automatic Control, vol. 48, no. 9, pp. 1509-1525, 2003.

[3] T. Hu, A. R. Teel, and L. Zaccarian, "Regional anti-windup compensation for linear systems with input saturation," in Proceedings of the American Control Conference, June 2005, pp. 3397-3402 vol. 5.

[4] C. Pittet, S. Tarbouriech, and C. Burgat, "Stability regions for linear systems with saturating controls via circle and Popov criteria," in Proceedings of the 36th IEEE Conference on Decision and Control, vol. 5, Dec 1997, pp. 4518-4523 vol.5.

[5] Y. Y. Cao and Z. Lin, "An anti-windup design for polytopic systems by a parameter-dependent Lyapunov function approach," in International Conference on Control and Automation, vol. 1, June 2005, pp. 541546 Vol. 1.

[6] F. Garelli, R. J. Mantz, and H. Battista, Advanced control for constrained processes and systems. The Institution of Engineering and Technology, 2011.

[7] F. Garelli, P. Camocardi, and R. J. Mantz, "Variable structure strategy to avoid amplitude and rate saturation in pitch control of a wind turbine," International Journal of Hydrogen Energy, vol. 35, no. 11, pp. 5869-5875, 2010.

[8] K. Aström and T. Hägglund, PID Controllers: Theory, Design and Tuning. Instrument Society of America, 1995.

[9] I. Boiko, Non-parametric tuning of PID controllers. Springer, 2013.

[10] C. C. Yu, Autotuning of PID controllers: a relay feedback approach. Springer Science \& Business Media, 2006.

[11] J. T. Pukrushpan, A. G. Stefanopoulou, and H. Peng, Control of fuel cell power systems: principles, modeling, analysis and feedback design. Springer Science \& Business Media, 2004.

[12] Y. Shtessel, C. Edwards, L. Fridman, and A. Levant, Sliding Mode Control and Observation, ser. Control Engineering. Springer New York, 2013. 